\title{
The Actinobacterial mce Operon: Structure and Functions
}

\author{
M. V. Zaychikova ${ }^{a, *}$ and V. N. Danilenko ${ }^{a}$ \\ ${ }^{a}$ Vavilov Institute of General Genetics, Russian Academy of Sciences, Moscow, 117971 Russia \\ *e-mail: marinaz15@yandex.ru \\ Received January 17, 2020; revised January 17, 2020; accepted January 17, 2020
}

\begin{abstract}
The results of studies on mce operons of the causative agent Mycobacterium tuberculosis and actinobacteria are summarized. Mce transporters belong to a group of cell-wall proteins. The genome of M. tuberculosis contains four mce operons that encode complexes, each of which consists of two integral membrane proteins (YrbEB) followed by six other Mce proteins (MceA-F). These proteins are functionally similar to ABC transporters. Despite their significant role in the pathogenesis of M. tuberculosis infection, Mce proteins remain poorly studied due to their complex structure and operon regulation. However, a number of their functions have been characterized; they include the penetration of host cells by M. tuberculosis and its survival, as well as the transport of cholesterol and mycolic acids, which are pathogenicity factors of no small importance. The expression of $m c e$ operons depends on many factors, such as the growth phase, the culture medium, and the localization of M. tuberculosis infection. Today, strains of $M$. tuberculosis and M. bovis with deleted mce operons are considered candidates for the development of new attenuated vaccines that might one day replace the $M$. bovis BCG vaccine, which is no longer deemed strong enough to hold back the tuberculosis epidemic.
\end{abstract}

Keywords: virulence Mycobacterium tuberculosis, mce operon, actinobacteria, ABC transporters

DOI: $10.1134 /$ S2079086420060079

\section{INTRODUCTION}

Tuberculosis is currently one of the most common infectious diseases and one of the ten leading causes of death in the world (Global..., 2019). Of particular danger is the increase in the proportion of strains with multidrug and wide drug resistance and increased virulence (Ribeiro et al., 2014; Lange et al., 2018). Virulence refers to the ability of a pathogen to infect a host organism. It is determined by a number of factors and depends both on the host, including its immunoresponse, and on the pathogen itself (Marimani et al., 2018). The clinical manifestations and epidemiology of tuberculosis are thus determined by the balance between the host immune system and pathogen virulence systems. The genome of Mycobacterium tuberculosis includes more than 300 genes that encode various virulence factors involved at different stages of the colonization of the host organism, including adhesion, cellular incorporation, avoidance of the immune system response, etc. (Forrellad et al., 2013; Prozorov et al., 2014). These include genes that determine the biosynthesis of mycolic acids, translational regulators of WhiB, serine-threonine protein kinases, genes encoding type-VII secretion systems, genes of toxinantitoxin systems, etc. Their products are involved at various stages of the infection process and make it possible to colonize the mucous membrane of the host, to invade cells, to avoid the immune system response, to survive adverse conditions, etc. These pathogenicity factors include Mse proteins (Mammalian cell entry). A piece of DNA M. tuberculosis $450 \mathrm{bp} \mathrm{H37Ra,} \mathrm{which}$ gives Escherichia coli cells the ability to penetrate and survive in mammalian cells, was first identified in 1993 (Arruda et al., 1993). It is with this function, one of the first known, that the name of these proteins is associated. They are among the proteins of the cell wall and are involved in the penetration of $M$. tuberculosis cells in macrophages and their survival, as well as in the transport of cholesterol and mycolic acids. At present, Mce proteins have demonstrated presence of antigenic activity, which is of interest for their use as a potential basis for a genetically engineered vaccine (Ahmad et al., 2004).

\section{SPREAD OF mce OPERONS IN BACTERIA}

These proteins are not specific to M. tuberculosis and Mycobacterium tuberculosis complex. They are found in various pathogenic and nonpathogenic mycobacteria, such as $M$. intracellulare, $M$. smegmatis, and M. scrofulaceum, (Parker et al., 1995). In addition to mycobacteria, Mce operons are noted in the genomes of many free living and pathogenic microorganisms, including the families Intrasporangiaceae, and Streptomycetaceae, Nocardioidaceae (Casali and Riley, 2007; Mohn et al., 2008; Nakayama and Zhang-Akiyama, 2016; Hemati et al., 2019). 
According to Cole et al. (1998), mce modules are the result of tandem duplication; therefore, a number of mycobacteria have several mce copies. Thus, $N$. farcinica have two copies: mce 7 and mce8. In general, there is a tendency toward an increase in the number of $m c e$ operons in fast-growing mycobacteria as compared to slow-growing ones (Miller et al., 2004). M. smegmatis contains six mce operons, mce1-7. In this case, this microorganism has no mce 3 operon; the mce 1 operon is similar to that of $M$. tuberculosis, but it includes two additional genes (MSMEG_5902 and $M S M E G$ 5893), while there is an insert in operon $m c e 5$ between genes mce $5 A$ and mce $5 B$ (GarcíaFernández, 2017). A characteristic feature of operon $m c e 3$ is its decrease in a number of mycobacteria, such as $M$. bovis BCG, M. smegmatis, M. microti, and M. leprae (Ahmad et al., 2004). The latter is characterized by the presence of only one mce module in the genome. The presence of mce operons in nonpathogenic bacteria may indicate the existence of functions other than virulence in them. In particular, the participation of mce operons in cholesterol catabolism can explain their presence in free-living saprophytic bacteria, since there are steroid substances found in nature that bacteria can use as substrates for growth. At the same time, it was established that mce operons and their analogs, which are found in the genomes of certain pathogenic bacteria, participate in virulence. In Leptospira interrogans, the Mce protein participates in adhesion, allowing interaction with the receptors of the host cells (Cosate et al., 2016). Expression of $g l t T$ gene, an analog of the mce operon of Neisseria meningitidis, results in strains with high invasiveness and hypervirulence (Pagliarulo et al., 2004).

\section{MAIN STRUCTURAL AND FUNCTIONAL FEATURES OF Mce PROTEINS in Mycobacterium tuberculosis}

Proteins of Mce actinobacteria, including $M$. tuberculosisare ATP-binding cassette transporters. YrbE proteins are functionally homologous to permeases and have a region in the penultimate cytoplasmic loop that can serve as a site of interaction with ATPases, while other Mce are homologous to substrate-binding proteins (Casali and Riley, 2007). Between them, mce operons have a certain homology and are apparently the result of tandem duplication (Cole et al., 1998). All Mce proteins are characterized by a conserved TIGR00996 domain (IPR005693). It includes 304 amino acids, which is typical for actinomycetes.

Some of the mce operons in actinomycetes include the $m k l$ gene, which encodes an ATPase similar to ATPases of $\mathrm{ABC}$ transporters. A region in the cytoplasmic loop of YrbE that can serve as a site of interaction with Mkl-ATPase was identified. Mkl was found only in those actinomycetes with a genome containing genes that encode YrbE and Mce proteins
Operons often also include other genes that are functionally associated with mce or are involved in their regulation. Thus, the composition of mce 1 currently includes fadD5 (Rv0166), the intended function of which is to participate in the first stage of the degradation of fatty acids (Dunphy et al., 2010). In addition, before the operons mce1-3 located transcriptional regulators mceR.

\section{mce 1 Operon}

The mce 1 operon is present in all types of mycobacteria. The operon contains six genes (mce 1A-mce1F) that encode transporter proteins localized in the cell wall. (Chitale et al., 2001; Shimono et al., 2003; Stavrum et al., 2012). This operon is the most conservative of all mce operons and is the only one found in M. leprae, which is characterized by a strongly reduced gene (Forrellad et al., 2014). The mce1 operon has been studied for quite some time, but the results of these studies are often contradictory. Thus, the M. tuberculosis strains with knockout for $m c e 1$ demonstrate a hypervirulent phenotype when administered intravenously or intraperitoneally, while their virulence, conversely, is reduced upon intratracheal administration (Shimono et al., 2003; Gioffré et al., 2005).

The functions of the Mce1A protein were studied in greatest detail. This protein promotes the penetration of macrophages by $E$. coli cells and colloidal gold particles (Chitale et al., 2001). The functional domain of Inv3, which plays a key role in this, is a sequence comprising 22 amino acids (Lu et al., 2006).

Its functions are supposed to be in the transport of palmitic acid and mycolic acids, which are important virulence factors involved in the protection of the pathogen from the host immune system by inhibiting the inflammatory response in macrophages. Deletion of this operon leads to their accumulation in the extracellular space. The lipid profile of the cell also undergoes serious changes, including a decrease in the level of saccharolipids and glycerophospholipids and an increase in the level of alpha, methoxy, and ketomycolic acids. This is due to the fact that the deletion of the mce 1 operon leads to a decrease in the expression of genes involved in the transport and metabolism of lipids $m m p L 8, m m p L 10$, stfo, pks2 and papA2, the products of which are involved in the anti-inflammatory response. At the same time, the transcription of mmpL3, fasI, kasA, kasB, acpM, and RV3451, which are involved in the increase in the transport and metabolism of mycolic acids. Thus, deletion of the mcel operon, which changes the metabolism and composition of cell-wall lipids, affects the nature of the host's immunoresponse and determines the nature of the infection (Queiroz et al., 2015). In addition, this operon is expressed at the initial stage of infection, and its products are involved in the transition from the fast- to slow-growth phase (Beste et al., 2009). 


\section{mce2 Operon}

The mce 2 operon is present in all mycobacteria of the tuberculosis complex and is typical of the structure of $m c e$ operons and demonstrates significant homologous similarity with the mcel operon (Ahmad et al., 2005). A feature of $m c e 2$ is the presence of a gene fragment $R v 0590 A$ between $m c e 2 B$ and $m c e 2 C$ (Zhang and Xie, 2015). Mce 2 proteins are thought to be involved in the metabolism and import of sulfolipids (Marjanovic et al., 2011). Deletion of the mce 2 operon in the strain M. tuberculosis $\mathrm{H} 37 \mathrm{Rv}$ does not affect growth in vitro, but causes a weakening of the strain when laboratory animals are infected (Marjanovic et al., 2011). Deletion of this operon, as well as the gene phoP, in Mycobacterium bovis leads to a loss of virulence and the formation of an attenuated strain (García et al., 2016). The functions of individual operon proteins have not been studied to date, with the exception of Mce2E, which suppresses the innate immunoresponse of macrophages and promotes the proliferation of epithelial cells. Mce2E inhibits the activation of signaling pathways regulated by extracellular signal kinase (ERK) and Jun N-terminal kinase (JNK), which, in turn, is activated by mitogen-activated protein kinase (MAPK), which leads to a decrease in the expression of TNF and IL-6 in macrophages (Uchiya et al., 2013). In addition, Mce2E stimulates the cellular proliferation of epithelial pulmonary cells. Thus, Mce2E is a new, multifunctional virulence factor that regulates the host's immunoresponse (Qiang et al., 2016).

\section{mce3 Operon}

As previously mentioned, the mce 3 operon is absent in a number of mycobacteria, such as, M. bovis BCG, M. smegmatis, M. microti, and M. leprae (Ahmad et al., 2004, Zhang and Xie, 2011). It was established with the HeLa model cell line and latex beads that the Mce3A and Mce3E proteins that make up the operon are involved in invasion processes (ElShazly et al., 2007). An immunogenic reaction was shown for the proteins Mce3A, Mce3D, and Mce3E (Ahmad et al., 2004, Zhang and Xie, 2011). The expression of the mce3 operon is downregulated by $m c e 3 R$, which belongs to the tetR family. It, in turn, is under the regulation of its own product, Mce3R (Santangelo et al., 2008). These two genes are involved in phosphatidylinositol biosynthesis and lipid degradation, respectively (Santangelo et al., 2008).

It was shown that Mce3C participates in the adhesion to and penetration of macrophages, in which the Arg-Gly-Asp motif (RGD) is involved. Mce3C interacts with the $\beta 2$ integrins of the host, allowing penetration of the cell. Integrin binding to Mce3C stimulates $\beta 2$ integrin-dependent signal adapters and induces local actin rearrangement at the site of mycobacterial invasion (Zhang et al., 2018).
It was determined that the mce 3 operon is expressed in the cells of the pathogen exclusively upon infection of the lung tissue; no expression was found when the pathogen penetrated spleen cells. Thus, the expression of the mce 3 operon is tissue-specific (Kumar et al., 2003).

Mutations were detected in the genes of the mce 3 operon of $M$. tuberculosis. A thymine-to-cytosine mutation at position 44 in the $m c e 3 B$ gene is characteristic of isolates of the highly virulent F15/LAM4/KZN line, which is characterized by a high level of drug resistance. Another mutation in which adenine is replaced by cytosine at position 1229 of $m c e 3 F$ is characteristic of some isolates of the subline B0/W-148, which also has a high level of drug resistance and increased virulence. The subregion is named $\mathrm{B} 0 / \mathrm{N}-90$ (Mikheecheva et al., 2017).

\section{mce4 Operon}

The mce 4 operon is expressed in the stationary phase of mycobacterial growth culture in vitro or in the cells of laboratory animals. The degree of homology of this operon is quite high among various mycobacteria (Mitra et al., 2005; Saini et al., 2008; Timms et al., 2015).

As with Mce1A, the Mce4A protein gives E. coli cells the ability to penetrate HeLa cells (Zhang and Xie, 2011). Deletion of the mce 4 operon leads to a significant reduction in virulence Mycobacterium tuberculosis (Zhang and Xie, 2011; Khan et al., 2016). For Mce4F, participation in virulence (i.e., invasion) was predicted via analysis in silico (Rodríguez et al., 2015). However, the main function of these proteins is to participate in the catabolism of cholesterol. This is part of the membranes of the host cells, which mycobacteria are used as a source for their own development.

The Mce4E protein inhibits macrophage activity, disrupts phagocytosis, and thus plays an important role in the interaction of the host with the pathogen, since alveolar macrophages have an antigen-presenting function and are involved in the production of cytokines and TNF- $\alpha$ (Xu et al., 2008).

Thus, Mce proteins are involved in the development of the infectious process via inhibition of the activity of alveolar macrophages and the host immune system interactions, and they participate in the formation of granulomas (Marjanovic et al., 2010). Being functionally and structurally similar to ABC transporters, they are involved in the transport of lipids, which are virulence factors (Hemati et al., 2019). Mycobacterium tuberculosis Mce proteins of can help pierce the outer lipid layer and can form a channel through this lipid bilayer.

In, Mycobacterium avium subsp. paratuberculosis, Mce proteins are a key factor in the interaction with the host immune system (Ghosh et al., 2012). 


\section{FEATURES OF THE EXPRESSION \\ OF mce OPERONS AT DIFFERENT STAGES OF GROWTH}

In the study of the expression of these operons, it was initially found that mce 1 expression is observed at the exponential stage of culture growth, which almost disappears in the stationary phase when $m c e 4$ begins to be expressed. The mce 2 and mce 3 operons were expressed exclusively in the tissues of infected animals in the stationary phase (Kumar et al., 2003). It was subsequently found in 2016 that mce 1 continues to be expressed in the stationary phase (Singh et al., 2016). Obviously, the difference from the results of Kumar et al. (2003) are methodological differences (Kumar et al., 2003; Singh et al., 2016). The expression of the mce operon occurs mainly in the stationary growth phase, in conditions of depleted nutrients and the accumulation of metabolic products. This can be interpreted as the participation of mce in survival in adverse conditions, including macrophages. This is also indicated by the fact that the expression of genes such as Rv3843c, ponA1, uvrA, Rv3070, pks2, ctpV, prrA, and sigE is observed in the stationary growth phase, which is characteristic of the development of M. tuberculosis in vivo in the tissues of infected laboratory animals (Singh et al., 2016).

\section{CONCLUSIONS}

The mce operons of M. tuberculosis undoubtedly play an extremely important role in pathogenesis and virulence. Their functions are very diverse and consist of the transport of other virulence factors, including mycolic acids and lipids, and direct interaction with the host cells and its immune systems. Although this group of genes has been studied for two decades, their functions and role in the pathogenesis of M. tuberculosis are not completely clear. The expression of mce operons depends on the growth stage, the used model systems (in vitro or in vivo), and the localization of the infectious process in the laboratory animals. In addition, the presence of single-nucleotide polymorphisms, which gradually appeared and were accumulated during evolution, is characteristic of mce genes. A significant part of the polymorphisms leading to the replacement of the amino acid can affect the structure of the protein product, changing its properties, including the antigenic activity (Ahmad et al., 2004; Mikheecheva et al., 2017). Analysis of in silico mutations in the genes of the mce 1 and mce 4 operons in M. tuberculosis made it possible for some of them to predict a significant effect on the structure and antigenic properties (Pasricha et al., 2011). These genes have no analogs in the human genome, which makes them ideal candidates for vaccine development. In addition, the mce operon is absent in M. bovis; therefore, prior $\mathrm{BCG}$ vaccination will not affect the immune response.
In this regard, this group of genes is of undoubted interest for research and could be used create new genetically engineered vaccines.

\section{FUNDING}

This work was supported by a grant from the Russian National Fund, project no. 17-75-20060 "Search for biotargets of potential anti-tuberculosis drugs of the azolo[1,2,4,5]tetrazine class" (2017-2019).

\section{COMPLIANCE WITH ETHICAL STANDARDS}

Conflict of interests. The authors declare that they have no conflicts of interest.

Statement on the welfare of humans or animals. This article does not contain any studies involving animals performed by any of the authors.

\section{REFERENCES}

Ahmad, S., El-Shazly, S., Mustafa, A.S., et al., Mammalian cell-entry proteins encoded by the mce 3 operon of Mycobacterium tuberculosis are expressed during natural infection in humans, Scand. J. Immunol., 2004, vol. 60, no. 4, pp. 382-391.

Ahmad, S., El-Shazly, S., Mustafa, AS., et al., The six mammalian cell entry proteins (Mce3A-F) encoded by the $m c e 3$ operon are expressed during in vitro growth of Mycobacterium tuberculosis, Scand. J. Immunol., 2005, vol. 62 , no. 1, pp. 16-24.

Arruda, S., Bomfim, G., Knights, R., et al., Cloning of an $M$. tuberculosis DNA fragment associated with entry and survival inside cells, Science, 1993, vol. 261, no. 5127, pp. 1454-1457.

Beste, D.J.V., Espasa, M., Bonde, B., et al., The genetic requirements for fast and slow growth in mycobacteria, PLoS One, 2009, vol. 4, p. e5349.

Casali, N. and Riley, L.W., A phylogenomic analysis of the actinomycetales mce operons, BMC Genomics, 2007, vol. 26, no. 8, p. 60.

Chitale, S., Ehrt, S., Kawamura, I., et al., Recombinant Mycobacterium tuberculosis protein associated with mammalian cell entry, Cell. Microbiol., 2001, vol. 3, no. 4, pp. 247-254

Cole, S.T., Brosch, R., Parkhill, J., et al., Deciphering the biology of Mycobacterium tuberculosis from the complete genome sequence, Nature, 1998, vol. 393, no. 6685 , pp. 537-544.

Cosate, M.R., Siqueira, G.H., de Souza, G.O., et al., Mammalian cell entry (Mce) protein of Leptospira interrogans binds extracellular matrix components, plasminogen and $\beta 2$ integrin, Microbiol. Immunol., 2016, vol. 60, no. 9, pp. 586-598.

Dunphy, K.Y., Senaratne, R.H., Masuzawa, M., et al., Attenuation of Mycobacterium tuberculosis functionally disrupted in a fatty acyl-coenzyme A synthetase gene fadD5, J. Infect. Dis., 2010, vol. 201, pp. 1232-1239.

El-Shazly, S., Ahmad, S., Mustafa, A.S., et al., Internalization by HeLa cells of latex beads coated with mammalian cell entry (Mce) proteins encoded by the $m c e 3$ op- 
eron of Mycobacterium tuberculosis, J. Med. Microbiol., 2007, vol. 56, no. 9, pp. 1145-1151.

Forrellad, M.A., Klepp, L.I., Gioffre, A., et al., Virulence factors of the Mycobacterium tuberculosis complex, Virulence, 2013, vol. 4, no. 1, pp. 3-66.

Forrellad, M.A., McNeil, M., Santangelo, M.L., et al., Role of the Mce 1 transporter in the lipid homeostasis of Mycobacterium tuberculosis, Tuberculosis (Edinburgh), 2014, vol. 94, no. 2, pp. 170-177.

García, E., Bianco, M.V., Blanco, F.C., et al., Mycobacterium bovis deleted in mce 2 and phoP loci protects C57BL/6 mice against tuberculosis, J. Infect. Dev. Countries, 2016, vol. 10, no. 8, pp. 892-894.

García-Fernández, J., Papavinasasundaram, K., Galán, B., et al., Molecular and functional analysis of the mce 4 operon in Mycobacterium smegmatis, Environ. Microbiol., 2017, vol. 19, no. 9, pp. 3689-3699.

Ghosh, P., Hsu, C., Alyamani, E.J., et al., Genome-wide analysis of the emerging infection with Mycobacterium avium subspecies paratuberculosis in the Arabian camels (Camelus dromedarius), PLoS One, 2012, vol. 7, no. 2, p. e31947.

Gioffré, A., Infante, E., Aguilar, D., et al., Mutation in mce operons attenuates Mycobacterium tuberculosis virulence, Microb. Infect. Inst. Pasteur, 2005, vol. 7, pp. 325-334.

Global Tuberculosis Report 2017, Geneva: World Health Org., 2019.

Hemati, Z., Derakhshandeh, A., Haghkhah, M., et al., Mammalian cell entry operons; novel and major subset candidates for diagnostics with special reference to $M y$ cobacterium avium subspecies paratuberculosis infection, Vet. Quart., 2019, vol. 39, no. 1, pp. 65-75.

Khan, S., Islam, A., Hassan, M.I., et al., Purification and structural characterization of Mce4A from Mycobacterium tuberculosis, Int. J. Biol. Macromol., 2016, vol. 93, pp. 235-241.

Kumar, A., Bose, M., and Brahmachari, V., Analysis of expression profile of mammalian cell entry (mce) operons of Mycobacterium tuberculosis, Infect. Immun., 2003, vol. 71, no. 10, pp. 6083-6087.

Lange, C., Chesov, D., Heyckendorf, J., et al., Drug-resistant tuberculosis: an update on disease burden, diagnosis and treatment, Respirology, 2018, vol. 23, no. 7, pp. 656-673.

Lu, S., Tager, L.A., Chitale, S., and Riley, L.W., A cellpenetrating peptide derived from mammalian cell uptake protein of Mycobacterium tuberculosis, Anal. Biochem., 2006, vol. 353, no. 1, pp. 7-14.

Marimani, M., Ahmad, A., and Duse, A., The role of epigenetics, bacterial and host factors in progression of Mycobacterium tuberculosis infection, Tuberculosis (Edinburgh), 2018, vol. 113, pp. 200-214.

Marjanovic, O., Iavarone, A.T., and Riley, L.W., Sulfolipid accumulation in Mycobacterium tuberculosis disrupted in the mce 2 operon, J. Microbiol., 2011, vol. 49, no. 3, pp. 441-447.

Marjanovic, O., Miyata, T., Goodridge, A., et al., Mce2 operon mutant strain of Mycobacterium tuberculosis is attenuated in C57BL/6 mice, Tuberculosis (Edinburgh), 2010, vol. 90, no. 1, pp. 50-56.
Mikheecheva, N.E., Zaychikova, M.V., Melerzanov, A.V., et al., A nonsynonymous SNP catalog of Mycobacterium tuberculosis virulence genes and its use for detecting new potentially virulent sublineages, Gen. Biol. Evol., 2017, vol. 9, no. 4, pp. 887-899.

Miller, C.D., Hall, K., Liang, Y.N., et al., Isolation and characterization of polycyclic aromatic hydrocarbondegrading Mycobacterium isolates from soil, Microb. Ecol., 2004, vol. 48, no. 2, pp. 230-238.

Mitra, D., Saha, B., Das, D., et al., Correlating sequential homology of Mce1A, Mce2A, Mce3A and Mce4A with their possible functions in mammalian cell entry of $M y$ cobacterium tuberculosis performing homology modeling, Tuberculosis (Edinburgh), 2005, vol. 85, nos. 5-6, pp. 337-345.

Mohn, W.W., van der Geize, R., Stewart, G.R., et al., The actinobacterial mce 4 locus encodes a steroid transporter, J. Biol. Chem., 2008, vol. 283, no. 51, pp. 3536835374.

Nakayama, T. and Zhang-Akiyama, Q.M., pqiABC and yebST, putative mce operons of Escherichia coli, encode transport pathways and contribute to membrane integrity, J. Bacteriol., 2016, vol. 199, no. 1, p. e00606-16.

Pagliarulo, C., Salvatore, P., De Vitis, L.R., et al., Regulation and differential expression of $g d h A$ encoding NADP-specific glutamate dehydrogenase in Neisseria meningitidis clinical isolates, Mol. Microbiol., 2004, vol. 51, no. 6, pp. 1757-1772.

Parker, S.L., Tsai, Y.L., and Palmer, C.J., Comparison of PCR-generated fragments of the mce gene from Mycobacterium tuberculosis, M. avium, M. intracellulare, and M. scrofulaceum, Clin. Diagn. Lab. Immunol., 1995, vol. 2, no. 6, pp. 770-775.

Pasricha, R., Chandolia, A., Ponnan, P., et al., Single nucleotide polymorphism in the genes of mce 1 and mce 4 operons of Mycobacterium tuberculosis: analysis of clinical isolates and standard reference strains, $B M C M i-$ crobiol., 2011, vol. 11, p. 41.

Prozorov, A.A., Fedorova, I.A., Bekker, O.B., et al., The virulence factors of Mycobacterium tuberculosis: genetic control, new conceptions, Russ. J. Genet., 2014, vol. 50, no. 8 , pp. $775-797$.

Qiang, L., Wang, J., Zhang, Y., et al., Mycobacterium tuberculosis Mce2E suppresses the macrophage innate immune response and promotes epithelial cell proliferation, Cell Mol. Immunol., 2019, vol. 16, no. 4, pp. 380391.

Queiroz, A., Medina-Cleghorn, D., Marjanovic, O., et al., Comparative metabolic profiling of mcel operon mutant vs wild-type Mycobacterium tuberculosis strains, Pathog. Dis., 2015, vol. 73, no. 8, art. ID ftv066

Ribeiro, S.C., Gomes, L.L., Amaral, E.P., et al., Mycobacterium tuberculosis strains of the modern sublineage of the Beijing family are more likely to display increased virulence than strains of the ancient sublineage, J. Clin. Microbiol., 2014, vol. 52, pp. 2615-2624.

Rodríguez, D.C., Ocampo, M., Varela, Y., et al., Mce4F Mycobacterium tuberculosis protein peptides can inhibit invasion of human cell lines, Pathog. Dis., 2015, vol. 73, no. 3, art. ID ftu020.

Saini, N.K., Sharma, M., Chandolia, A., et al., Characterization of Mce4A protein of Mycobacterium tuberculo- 
sis: role in invasion and survival, BMC Microbiol., 2008, vol. 8, no. 1, p. 200.

Santangelo, M.P., Blanco, F.C., Bianco, M.V., et al., Study of the role of Mce3R on the transcription of mce genes of Mycobacterium tuberculosis, BMC Microbiol., 2008, vol. 8 , no. 1 , p. 38 .

Shimono, N., Morici, L., Casali, N., et al., Hypervirulent mutant of Mycobacterium tuberculosis resulting from disruption of the mcel operon, Proc. Natl. Acad. Sci. U.S.A., 2003, vol. 100, pp. 15918-1592.

Singh, P., Katoch, V.M., Mohanty, K.K., et al., Analysis of expression profile of mce operon genes (mce 1, mce2, mce 3 operon) in different Mycobacterium tuberculosis isolates at different growth phases, Indian J. Med. Res., 2016, vol. 143, no. 4, pp. 487-494.

Stavrum, R., Valvatne, H., Stavrum, A.-K., et al., Mycobacterium tuberculosis Mce1 protein complex initiates rapid induction of transcription of genes involved in substrate trafficking, Genes Immun., 2012, vol. 13, no. 6, pp. 496-502.
Timms, V.J., Hassan, K.A., Mitchell, H.M., et al., Comparative genomics between human and animal associated subspecies of the Mycobacterium avium complex: a basis for pathogenicity, BMC Genomics, 2015, vol. 16, p. 695.

Uchiya, K., Takahashi, H., Yagi, T., et al., Comparative genome analysis of Mycobacterium avium revealed genetic diversity in strains that cause pulmonary and disseminated disease, PLoS One, 2013, vol. 8, no. 8, p. e71831.

Xu, G., Li, Y., Yang, J., et al., Mycobacterium bovis Mce4E protein may play a role in modulating cytokine expression profile in alveolar macrophage, Int. J. Tuberc. Lung Dis., 2008, vol. 12, pp. 664-669.

Zhang, F. and Xie, J.P., Mammalian cell entry gene family of Mycobacterium tuberculosis, Mol. Cell Biochem., 2011, vol. 352, nos. 1-2, pp. 1-10.

Zhang, Y., Li, J., Li, B., et al., Mycobacterium tuberculosis Mce3C promotes mycobacteria entry into macrophages through activation of $\beta 2$ integrin-mediated signaling pathway, Cell Microbiol., 2018, vol. 20, no. 2. https://doi.org/10.1111/cmi.12800 